\title{
Effect of Interleukin-11 with and without Granulocyte Colony-Stimulating Factor on In Vivo Neonatal Rat Hematopoiesis: Induction of Neonatal Thrombocytosis by Interleukin-11 and Synergistic Enhancement of Neutrophilia by Interleukin-11 + Granulocyte Colony-Stimulating Factor $^{1}$
}

\author{
MITCHELL S. CAIRO, J. MICHAEL PLUNKETT, ANNA NGUYEN, PAUL SCHENDEL, AND \\ CARMELLA VAN DE VEN \\ Genetics Institute, Cambridge, Massachusetts 02140; and Children's Hospital of Orange County, \\ Orange, California 92668
}

\begin{abstract}
IL-11, a new hematopoietic cytokine isolated from primate stromal cells (PU-34), has been shown to act synergistically with IL-3 to induce proliferation of early hematopoietic stem cells and induce in vitro CFU-MEG proliferation. We hypothesize that recombinant human (rh)IL-11 alone or in combination with granulocyte colonystimulating factor (G-CSF) might modulate newborn in vivo granulopoiesis and thrombopoiesis. Newborn Sprague-Dawley rats were given $14 \mathrm{~d}$ of intraperitoneal rhIL$11(0-250 \mu \mathrm{g} / \mathrm{kg} \times 14 \mathrm{~d}), \mathrm{rhIL}-11(250 \mu \mathrm{g} / \mathrm{kg})+\mathrm{rhG}-\mathrm{CSF}$ $(5 \mu \mathrm{g} / \mathrm{kg}$ simultaneously $\times 14 \mathrm{~d})$, rhIL-11 $\times 7 \mathrm{~d}$ followed by G-CSF $\times 7$ d, G-CSF $\times 14$ d, PBS/human serum albumin $\times 7 \mathrm{~d}$ followed by G-CSF $\times 7 \mathrm{~d}$, or PBS/human serum albumin $\times 14 \mathrm{~d}$. rhIL-11 alone had no effect on the circulating hematocrit or absolute neutrophil count. There was, however, a significant increase in the circulating platelet count after rhIL-11 (100 and $250 \mu \mathrm{g} / \mathrm{kg}$ ) versus PBS/ human serum albumin (d 13: $1241 \pm 54,1262 \pm 58$ versus $\left.939 \pm 38 \mathrm{k} / \mathrm{mm}^{3} ; p=0.01\right)$. Sequential and simultaneous IL-11 + G-CSF caused a significant increase in the marrow neutrophil reserve and the circulating absolute neutrophil count above that observed when G-CSF alone was administered. IL-11 \pm G-CSF, however, failed to reduce the 96h mortality rate during experimental group B streptococcal sepsis. These data suggest that IL-11 alone results in a significant elevation in the blood platelet concentration and, in combination with G-CSF, induces an increase in in vivo neonatal rat myelopoiesis. This novel cytokine may have the potential to decrease the morbidity associated with cytopenias in the newborn. (Pediatr Res 34: 56-61, 1993)
\end{abstract}

\section{Abbreviations}

G-CSF, granulocyte colony-stimulating factor $\mathrm{CSF}$, colony stimulating factor

Received December 21, 1992; accepted February 26, 1993.

Correspondence: Mitchell S. Cairo, M.D., Director, Hematology/Oncology Research and Bone Marrow Transplantation, Children's Hospital of Orange County, 455 S. Main St., Orange, CA 92668.

Supported by grants from the Pediatric Cancer Research Foundation, the Walden W. and Jean Young Shaw Foundation, and the CHOC Research and Education Foundation.

' Presented at the Western Society for Pediatric Research, Carmel, CA, February

\author{
rh, recombinant human \\ rr, recombinant rat \\ $\mathrm{SCF}$, stem cell factor \\ HSA, human serum albumin \\ NSP, neutrophil storage pool \\ NPP, neutrophil proliferative pool \\ CFU, colony-forming unit \\ GEMM, granulocyte, erythroid, monocyte, megakaryocyte \\ GM, granulocyte macrophage \\ BM, bone marrow \\ MEG, megakaryocyte
}

Significant developmental immaturities in neonatal hematopoiesis have been identified to include defects in both myelopoiesis and thrombopoiesis. During states of increased demand, the newborn is predisposed to developing peripheral cytopenias, especially neutropenia and thrombocytopenia (1-3). Dysregulation of neonatal rat myelopoiesis has been demonstrated to be associated with both reduced CFU-GM progenitors and decreased BM NSP precursors (4-6). However, circulating pools of multipotent and unipotent myeloid progenitor cells (CFUGEMM and CFU-GM, respectively) are significantly elevated in both preterm and term infant cord blood compared with adult peripheral blood $(7,8)$.

We have previously demonstrated that single, sequential, and simultaneous administration of both early-acting and late-acting hematopoietic growth factors modulate both in vitro and in vivo neonatal hematopoiesis. Single-d, 7-, or 14-d administration of rhG-CSF in newborn rats induces peripheral neutrophilia and an increase in BM NSP and CFU-GM pools $(9,10)$. The sequential 7-d administration of both early and late-acting hematopoietic growth factors, rhIL-6, or rrSCF (early-acting) followed by rhG-CSF (late-acting), has been associated with a significant increase in neonatal rat peripheral neutrophilia and BM NSP and CFU-GM pools $(11,12)$. Similarly, simultaneous administration of an early lineage CSF (rrSCF) plus a lineage-specific CSF (rhG-CSF) also has resulted in a significant increase in both neonatal rat peripheral neutrophilia and BM NSP (12). We have also recently demonstrated that rhSCF is additive with both rhG- 
CSF or rhIL-3 to induce cord blood CFU-GM colony formation when incubated with isolated $\mathrm{CD} 34^{+}$progenitor cells (13). rhIL6 , however, has been the only hematopoietic growth factor that has been demonstrated to induce in vivo neonatal rat thrombocytosis (11).

Several hematopoietic growth factors have been identified to regulate the kinetics of early hematopoietic stem cells. SCF, IL6, and G-CSF have all been demonstrated to enhance IL-3dependent proliferation of early hematopoietic progenitor cells by shortening the $G_{0}$ period of dormant stem cells (14-16). Recently, a novel hematopoietic cytokine, IL-11, has been identified from a medium conditioned by primate BM stromal cells (PU-34) (17). IL-11 has also been demonstrated to act synergistically with IL-3 to induce the proliferation of early hematopoietic stem cells by shortening their $\mathrm{G}_{0}$ dormant period (18). Schibler et al. (19) demonstrated that IL-11 in vitro induces a dose-related increase in the cycling of fetal progenitor cells (erythroid blast-forming unit, CFU-GM, and CFU-erythroid, myeloid, megakarocytic). We hypothesize that rhIL-11 may also have profound modulating effects on in vivo neonatal rat hematopoiesis and host defense. In the present study, we determined the in vivo effects of rhIL-11 on neonatal rat hematopoiesis both alone and in combination with a lineage-specific CSF (rhG-CSF) and examined its modulating effect on the mortality rate during experimental group B streptococcal sepsis.

\section{MATERIALS AND METHODS}

rhIL-11. rhIL-11 was kindly supplied by Genetics Institute (Cambridge, MA). It was produced in Escherichia coli and purified to homogeneity. A biologic activity of $1.5 \times 10^{6} \mathrm{U} / \mathrm{mg}$ was determined by stimulation of the T10 cell line assay. The absence of measurable endotoxin contamination was demonstrated by the Limulus amebocyte lysate assay. rhIL-11 was diluted in PBS/.01\% HSA before injection for in vivo studies at a dose ranging from 0 to $250 \mu \mathrm{g} / \mathrm{kg}$ animal body weight.

$r h G-C S F$. rhG-CSF, kindly provided by Jeff Andresen (Amgen, Thousand Oaks, CA), was also prepared from $E$. coli to $95 \%$ purity before formulation in $0.025 \%$ HSA. The absence of measurable endotoxin contamination was demonstrated by the Limulus amebocyte lysate assay. Five $\mu \mathrm{g} / \mathrm{kg}$ purified $\mathrm{rhG}-\mathrm{CSF}$ were used (diluted with PBS, pH 7.4). A biologic activity of $2 \times$ $10^{3} \mathrm{U} / \mathrm{mg}$ was determined by granulocyte colony formation on human nonadherent bone marrow cells in semisolid media.

Animal inoculation. This study used litters of neonatal albino Sprague-Dawley rats (Bantin-Kingman Laboratories, Fremont, CA) $\leq 24 \mathrm{~h}$ old $(6-8 \mathrm{~g})$. Mothers of the litters were received $1 \mathrm{wk}$ before delivery and housed at the California State University, Fullerton, vivarium. Approval for this study was granted by the Animal Use Committee. The animals were maintained at constant room temperature, with water and rodent feed (Purina Chow) ad libitum. The site of injection was washed with Betadine solution (povidone-iodine, 10\%, Purdue Frederick, Norwalk, CT) and swabbed with $70 \%$ alcohol before each inoculation of growth factor. Animals were initially given daily injections of rhIL-11 for $14 \mathrm{~d}$ at concentrations of $1,10,100$, and $250 \mu \mathrm{g} / \mathrm{kg}$ body weight. Once a dose-response relationship was demonstrated, $250 \mu \mathrm{g} / \mathrm{kg}$ was chosen as the rhIL-11 concentration in the following combination experiments. Animals were then given injections of either rhIL-11, rhG-CSF $(5 \mu \mathrm{g} / \mathrm{kg})$, or simultaneous rhIL- 11 and rhG-CSF for $14 \mathrm{~d}$, PBS/HSA for $7 \mathrm{~d}$ followed by rhG-CSF for $7 \mathrm{~d}$, or of rhIL-11 for $7 \mathrm{~d}$ followed by rhG-CSF for $7 \mathrm{~d}$. Intraperitoneal injections of $0.100 \mathrm{~mL}$ were performed with a sterile tuberculin syringe fitted with a 27.5 -gauge needle. Control animals received $0.100-\mathrm{mL}$ injections of PBS $/ 0.01 \%$ HSA.

Quantification of circulating platelets, neutrophils, and BM myeloid pools. Twenty $\mu \mathrm{L}$ of free-flowing blood were collected by nicking the jugular vein with a sterile 25 -gauge needle. Samples were electronically counted (Serano-Baker Diagnostics, Allentown, PA) to determine platelet and white blood cell counts; blood smears were prepared and stained with Wright stain, and a 100- to 200-cell differential was performed. Femoral BM was removed surgically and aseptically on d 14, and neutrophil BM pools (NSP + NPP) were determined as previously described by Cairo et al. (10) (NSP = percentage of polymorphonuclear cells + bands + metamyelocytes; NPP $=$ percentage of blasts + promyelocytes + myelocytes) Absolute neutrophil counts were determined by the multiplication of the nucleated cell count times the percentage of neutrophils in the differentials.

$C F U-G M$ colony formation. BM was collected as previously described (10). After suspension in Hanks' balanced salt solution (Gibco Laboratories, Grand Island, NY), cells were cultured in methylcellulose media containing $0.8 \%$ methylcellulose, FCS (30\%), BSA $(1 \%)$, and mercaptoethanol $\left(10^{-4} \mathrm{M}\right)$ (Terry Fox Laboratories, Vancouver, BC, Canada). A total of $2 \times 10^{5}$ cells were stimulated by murine-spleen-cell-conditioned medium (1\%) (Terry Fox Laboratories) and erythropoietin $(4 \mathrm{U} / \mathrm{mL})$ (Amgen). Cell suspensions were plated in triplicate in $10 \times 35$ $\mathrm{mm}$ tissue culture dishes (Nunc, Denmark) and incubated at $5 \%$ $\mathrm{CO}_{2}, 37^{\circ} \mathrm{C}$, in a high humidity atmosphere. Cultures were evaluated at $10 \mathrm{~d}$ with aggregates of $>50$ cells considered "colonies." Myeloid colonies were plucked and stained, and specific lineage was confirmed. This agarose culture, however, was not optimal for CFU-MEG determination.

$C F U$ proliferative rate. Thymidine suicide was used to evaluate the proliferative rates of CFU-GM as previously described (10). Light-density bone marrow mononuclear cells from neonatal rats were placed in $50-\mathrm{mL}$ centrifuge tubes, nonradioactive thymidine and methyl- ${ }^{3} \mathrm{H}$-thymidine containing $0.1 \mathrm{mCi}$ (sp act, 75 $\mathrm{Ci} / \mathrm{mmol}$, ICN Radiochemicals, Irvine, CA) were added, tubes were incubated for $20 \mathrm{~min}$, and thymidine uptake was terminated by adding excess nonradioactive thymidine in ice-cold $\alpha$-minimum essential medium with FCS. The cell suspensions were then washed twice with cold thymidine media, and plated in methylcellulose as described above. Colonies were enumerated after $10 \mathrm{~d}$ of culture in a $5 \% \mathrm{CO}_{2}$ incubator at $37^{\circ} \mathrm{C}$. The thymidine suicide rate was determined by subtracting the average number of colonies formed per plate by cells exposed to ${ }^{3} \mathrm{H}$ thymidine from the average number of colonies per plate formed by cells exposed to nonradioactive thymidine, divided by the average colonies per plate from cells exposed to nonradioactive thymidine.

Organism. Group B streptococcus (type III, Norris, kindly provided by Dr. Gerald Fisher, Bethesda, MD) was isolated from an infected neonate and serotyped by the precipitin method using rabbit anti-sera. The organism was grown in Todd-Hewitt broth to logarithmic phase and then aliquoted and stored at $-70^{\circ} \mathrm{C}$ until use. Aliquots were thawed and allowed to grow to maximum-phase growth in fresh Todd-Hewitt broth. Organisms were then sedimented by centrifugation and washed three times in sterile PBS. Concentration of bacteria was determined by OD at $620 \mathrm{~nm}$, and a suspension of $2.5 \times 10^{8}$ organisms/g body weight $/ 0.100 \mathrm{~mL}$ was prepared for injection.

Experimental sepsis. After receiving rhIL-11, rhIL-11 + rhGCSF sequentially and simultaneously, rhG-CSF, PBS/HSA, or PBS/HSA/rhG-CSF for $14 \mathrm{~d}, 2.5 \times 10^{8}$ organisms/g of body weight of group B streptococcus was injected intraperitoneally into each group of animals. Survival was monitored for the next 120 h. Control animals received sham injections of PBS $/ 0.01 \%$ HSA.

Statistical analysis. All results are expressed as mean \pm SEM of four animals or multiple runs of three to five replicates of blood or BM samples. The probability of significant differences when comparing two treated groups was determined with the use of the unpaired $t$ test, whereas the probability of significant differences when examining multiple treatments was determined by using analysis of variance followed by the Student-NewmanKeuls multiple-range tests to define the unique subsets within the study. Statistical analyses were performed using the Biostat I 
statistical program (Sigma Soft, Placentia, CA) for the IBM personal computer. $p$ values $<0.05$ are considered significant.

\section{RESULTS}

The administration of rhIL-11 $(0-250 \mu \mathrm{g} / \mathrm{kg} \times 14 \mathrm{~d})$ did not induce any significant change in circulating absolute neutrophil count. D 14 absolute neutrophil count after 1, 10, 100, and 250 $\mu \mathrm{g} / \mathrm{kg}$ rhIL-11 $\times 14 \mathrm{~d}$ compared with PBS/HSA was $1240 \pm 193$ versus $872 \pm 84$ versus $1016 \pm 60$ versus $973 \pm 128$ versus 1368 $\pm 139(p=\mathrm{NS})$. Similarly, $14 \mathrm{~d}$ of rhIL-11 failed to induce a significant change in the circulating hematocrit: values were 21.7 \pm 0.3 versus $21.6 \pm 0.4$ versus $21.5 \pm 0.7$ versus $20.3 \pm 0.7$ versus $21.3 \pm 0.8(\%)(1$ versus 10 versus 100 versus $250 \mu \mathrm{g} / \mathrm{kg}$ versus $\mathrm{PBS} / \mathrm{HSA}$ ). However, $14 \mathrm{~d}$ of rhIL-11 (at the two highest doses) induced a significant increase in the circulating platelet count (Fig. 1) rhIL-11 did not induce any significant change in the circulating absolute lymphocyte, monocyte, eosinophil, or basophil counts compared with placebo control-treated animals.

The BM NSP, NPP, CFU-GM, and CFU-GM proliferative rates were also determined after $14 \mathrm{~d}$ of administration of rhIL11. There was a dose-related increase in the BM NSP after $14 \mathrm{~d}$ of rhIL-11 (Fig. 2). There was a small but significant increase in the BM NPP after $14 \mathrm{~d}$ of rhIL-11 versus PBS/HSA $(1109 \pm 142$ versus $657 \pm 166)[\mathrm{rhIL}-11(100 \mu \mathrm{g} / \mathrm{kg})$ versus $\mathrm{PBS} / \mathrm{HSA}](p=$ $\leq 0.05$ ). After $14 \mathrm{~d}$ of rhIL-11, there was a significant increase in the BM CFU-GM compared with placebo control-treated animals (BM CFU-GM: $68.0 \pm 3.6$ versus $46.3 \pm 2.4$ ) (colonies/2 $\times 10^{5}$ cells, $p=<0.01$ ); however, no significant difference was seen in the CFU-GM proliferative rate [BM CFU-GM proliferative rate: $45.2 \pm 2.1 \%$ versus $37.9 \pm 4.6 \%, p=\mathrm{NS}$; rhIL-11 $(250 \mu \mathrm{g} / \mathrm{kg})$ versus $\mathrm{PBS} / \mathrm{HSA}]$.

To determine the additive and/or synergistic effect of rhIL-11 \pm rhG-CSF on in vivo neonatal rat hematopoiesis, sequential and simultaneous studies were performed with rhIL-11 at the $250-\mu \mathrm{g} / \mathrm{kg} / \mathrm{d}$ dose. The sequential administration of rhIL-11 $\times 7$ $\mathrm{d}$ followed by $\mathrm{rhG}-\mathrm{CSF} \times 7 \mathrm{~d}$ demonstrated a significant increase in the circulating absolute neutrophil count during the $2 \mathrm{nd} w \mathrm{k}$ of therapy compared with PBS/HSA $\times 7 \mathrm{~d}$ followed by rhG-CSF $\times 7 \mathrm{~d}$ (Fig. 3). Similarly, but even more strikingly, $14 \mathrm{~d}$ of simultaneous administration of rhIL-11 + rhG-CSF synergistically and significantly induced an increase in the circulating

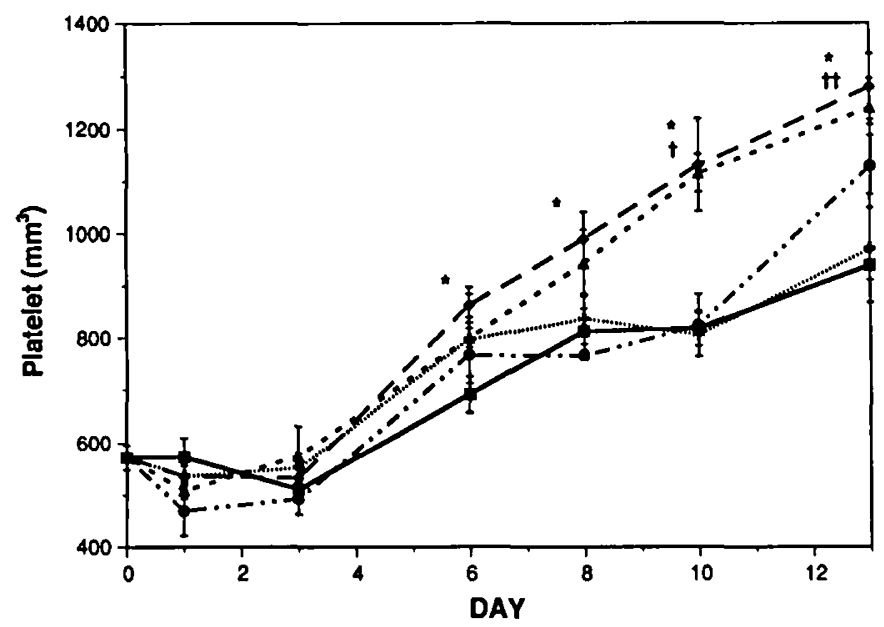

Fig. 1. Neonatal Sprague-Dawley rats ( $\leq 24 \mathrm{~h}$ ) received rhlL-11 or PBS/HSA for $14 \mathrm{~d}$ by daily intraperitoneal injection. Blood samples were electronically enumerated and platelet values obtained. Values are the mean \pm SEM of four animals. rhIL- $11(100 \mu \mathrm{g} / \mathrm{kg})$ vs PBS/HSA: $\uparrow, \mathrm{d} 10$ $(p<0.001)$; ††, d $13(p<0.05)$, rhIL-11 $(250 \mu \mathrm{g} / \mathrm{kg})$ vs PBS/HSA: *. d 6. 8, 10, and $13(p<0.005)$. $\cdots$, , IL-11 $(1 \mu \mathrm{g} / \mathrm{kg}) ;-\cdots-$, IL-11 $(10$ $\mu \mathrm{g} / \mathrm{kg}) ;-. .$. IL-11 $(100 \mu \mathrm{g} / \mathrm{kg}) ;-$, IL-11 $(250 \mu \mathrm{g} / \mathrm{kg}) ;-$. PBS/ HSA.

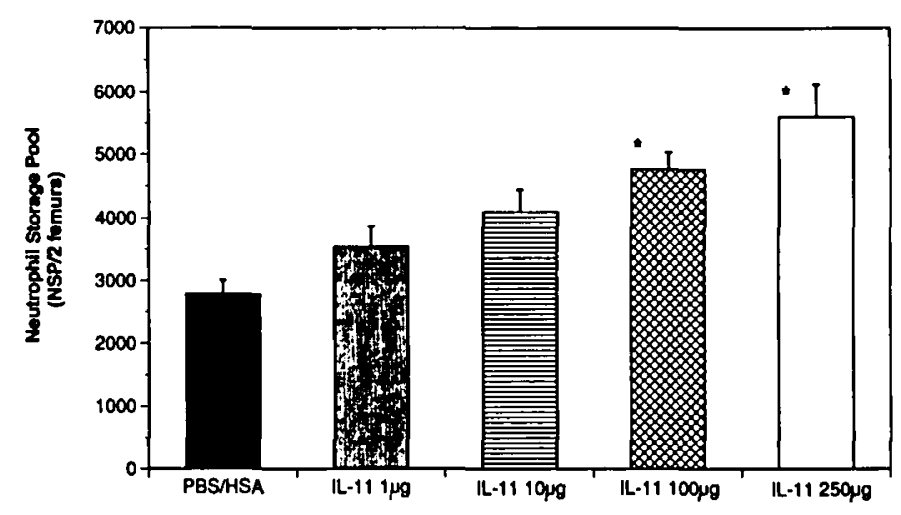

Fig. 2. Neonatal Sprague-Dawley rats $(\leq 24 \mathrm{~h})$ received rhIL-11 or PBS/HSA for $14 \mathrm{~d}$ by daily intraperitoneal injection. BM neutrophil storage pool was determined by aseptic removal of the femurs and flushing of the bone marrow into a known quanity of Hanks' balanced salt solution. Electronic cell counts were performed and a 500-cell differential was obtained on Wright's-stained cytospin preparations. Bars represent the cell number per two femurs (mean \pm SEM) of three replicates from pooled samples of four animals per treatment group. *, rhlL-11 $(100 \mu \mathrm{g} / \mathrm{kg}$ and $250 \mu \mathrm{g} / \mathrm{kg}) v s$ PBS/HSA: $p<0.001$.

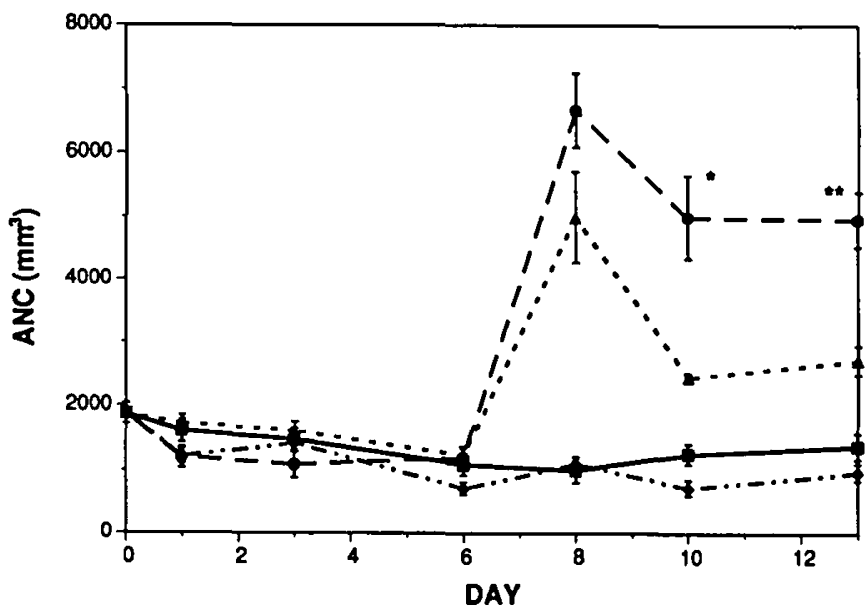

Fig. 3. Neonatal Sprague-Dawley rats $(\leq 24 \mathrm{~h})$ received rhIL-11 for $14 \mathrm{~d}$, rhIL-11 or PBS/HSA for $7 \mathrm{~d}$ followed by rhG-CSF for $7 \mathrm{~d}$, or PBS/ HSA for $14 \mathrm{~d}$ by daily intraperitoneal injection. Blood samples were electronically enumerated and a 100- to 200-cell differential was performed on Wright's-stained blood smears. Values are the mean \pm SEM of four animals. $A N C$, absolute neutrophil count. rhIL-11 + rhG-CSF $v s$ PBS/HSA + rhG-CSF: *, d $10(p<0.03),{ }^{* *}, \mathrm{~d} 13(p<0.05) .--$, IL$11 \times 7 \mathrm{~d}+\mathrm{G}-\mathrm{CSF} \times 7 \mathrm{~d} ;-\cdots-$, IL-11 $\times 14 \mathrm{~d} ; \cdots,-$, PBS $\times 7 \mathrm{~d}+\mathrm{G}-$ $\mathrm{CSF} \times 7 \mathrm{~d} ;-$ PBS/HSA $\times 14 \mathrm{~d}$.

absolute neutrophil count during the 2 nd wk of therapy ( $p=$ 0.02) (Fig. 4).

The increase in the circulating platelet count was entirely dependent on $14 \mathrm{~d}$ of administration of rhIL-11. In the groups of animals that received $14 \mathrm{~d}$ of rhIL-11 alone or simultaneously with rhG-CSF, there was a significantly higher circulating platelet count compared with those treated with either rhIL-11 $\times 7 \mathrm{~d}$ followed by rhG-CSF $\times 7 \mathrm{~d}$, G-CSF alone $\times 14 \mathrm{~d}$, or PBS/HSA $\times 14 \mathrm{~d}$ (Fig. 5). G-CSF had no effect on circulating platelet count. There was no significant change in the combination studies relative to the circulating hematocrit and absolute lymphocyte, basophil, monocyte, and eosinophil counts.

Both the sequential and simultaneous administration of rhlL11 and rhG-CSF induced a significant increase in the BM NSP compared with control-treated animals or rhG-CSF alone (Fig. 6). There was, however, no significant difference in the BM NPP when rhIL-11 was used either sequentially or simultaneously with rhG-CSF versus rhG-CSF alone or PBS-HSA-treated ani- 


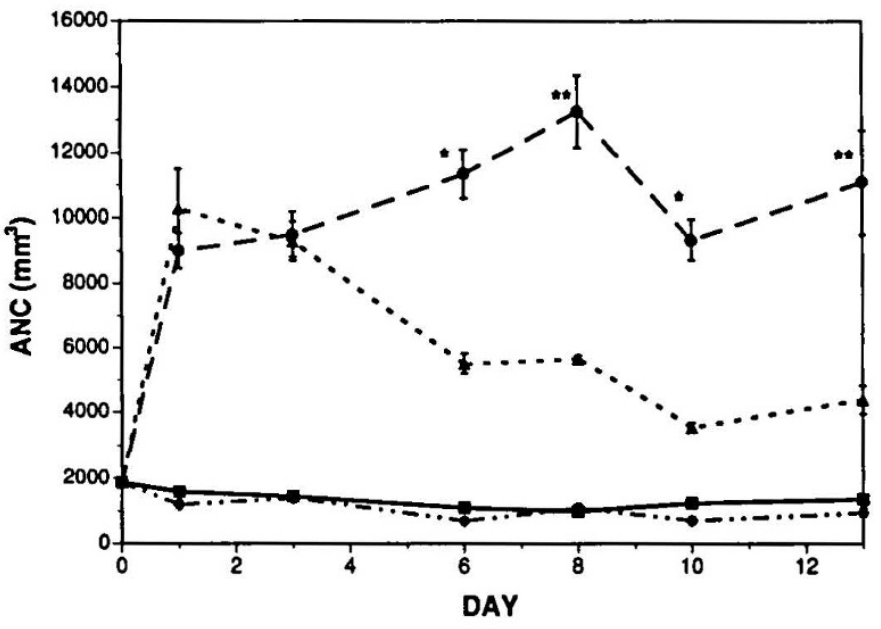

Fig. 4. Neonatal Sprague-Dawley rats $(\leq 24 \mathrm{~h})$ received rhlL-11 for $14 \mathrm{~d}$. rhIL-11 + rhG-CSF for $14 \mathrm{~d}$, or rhG-CSF or PBS/HSA for $14 \mathrm{~d}$ by daily intraperitoneal injection. Blood samples were electronically enumerated and a 100- to 200-cell differential was performed on Wright'sstained blood smears. Values are the mean \pm SEM of four animals. $A N C$, absolute neutrophil count. rhIL-11 + rhG-CSF is G-CSF: *. d 6, 10 ( $p$ $<0.005)$ : ${ }^{* *}$. d $8.13(p<0.02),--$ IL-11 + G-CSF $\times 14 \mathrm{~d}:-\cdots$. IL-11 $\times 14 \mathrm{~d}:-\cdots$ G-CSF $\times 14 \mathrm{~d}:-$. PBS/HSA $\times 14 \mathrm{~d}$.

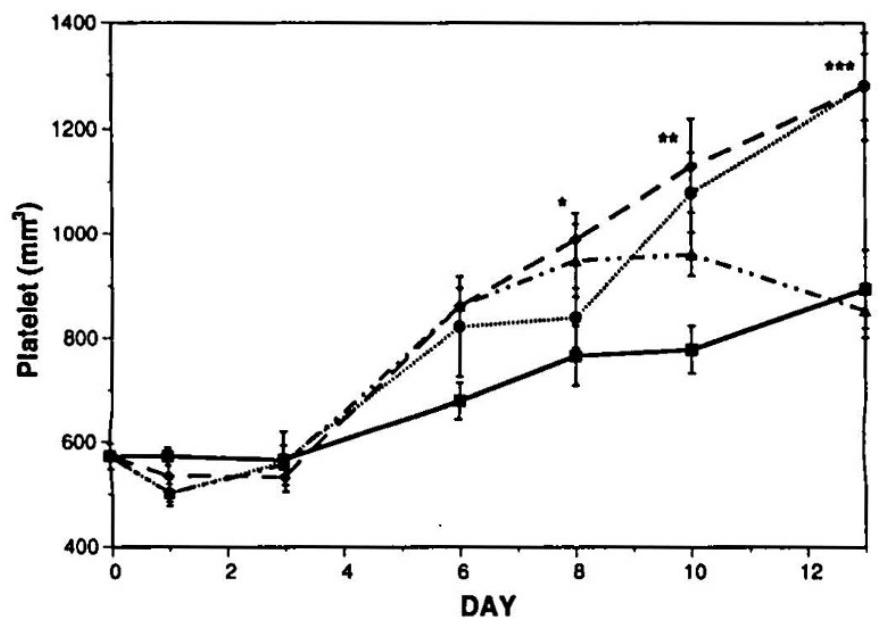

Fig. 5. Neonatal Sprague-Dawley rats $(\leq 24 \mathrm{~h})$ received rhlL-11 for $14 \mathrm{~d}$. rhIL-11 for $7 \mathrm{~d}$ followed by rhG-CSF for $7 \mathrm{~d}$, rhIL-11 and rhGCSF for $14 \mathrm{~d}$, or PBS/HSA for $14 \mathrm{~d}$ by daily intraperitoneal injection. Blood samples were electronically enumerated and platelet values obtained. Values are the mean \pm SEM of four animals. rhIL-11 $\times 7 \mathrm{~d}+$ G-CSF $\times 7 \mathrm{~d}$ is PBS/HSA: *, d $8(p<0.05){ }^{* *}$, d $10(p<0.02)$. rhlL$11+\mathrm{G}-\mathrm{CSF} \times 14 \mathrm{~d}$ is PBS/HSA: ${ }^{* *}, \mathrm{~d} 10(p<0.001):^{* * *}, \mathrm{~d} 13(p<$ $0.02) .-\cdots-$ IL-11 $\times 7 \mathrm{~d}+\mathrm{G}-\mathrm{CSF} \times 7 \mathrm{~d} ; \cdots$, , IL-11 + G-CSF $\times 14$ $\mathrm{d}:-$. IL- $11 \times 14 \mathrm{~d}:-$ PBS/HSA $\times 14 \mathrm{~d}$.

mals (data not shown). The sequential and simultaneous treatment of rhIL-11 + rhG-CSF also induced a significant increase in the BM CFU-GM proliferative rate compared with G-CSF or control-treated animals [ $49 \pm 3.9 \%$ rhIL-11 (sequential) + GCSF, $p=0.05$, and $56.4 \pm 3.2 \%$ rhIL- 11 (simultaneous) $+\mathrm{G}-$ CSF, $p=0.01$, versus $31.7 \pm 4.1 \%$ G-CSF versus $35.3 \pm 3.5 \%$ PBS/HSA]. However, the BM CFU-GM was slightly increased but not significantly different in the sequential and simultaneous treatment with rhIL-11 and G-CSF [64.7 \pm 6.7 sequential, 64.7 \pm 10.1 simultaneous versus $54 \pm 3.5 \mathrm{G}-\mathrm{CSF}$ versus $46.3 \pm 2.4$ PBS/HSA colonies $/ 2 \times 10^{5} ; p=\mathrm{NS}$ ].

Lastly, we examined the prophylactic effect of $14 \mathrm{~d}$ of rhIL-11 alone or given sequentially or simultaneously with rhG-CSF in modulating the survival rate after inducing experimental group B streptococcal sepsis. There was a significant difference in

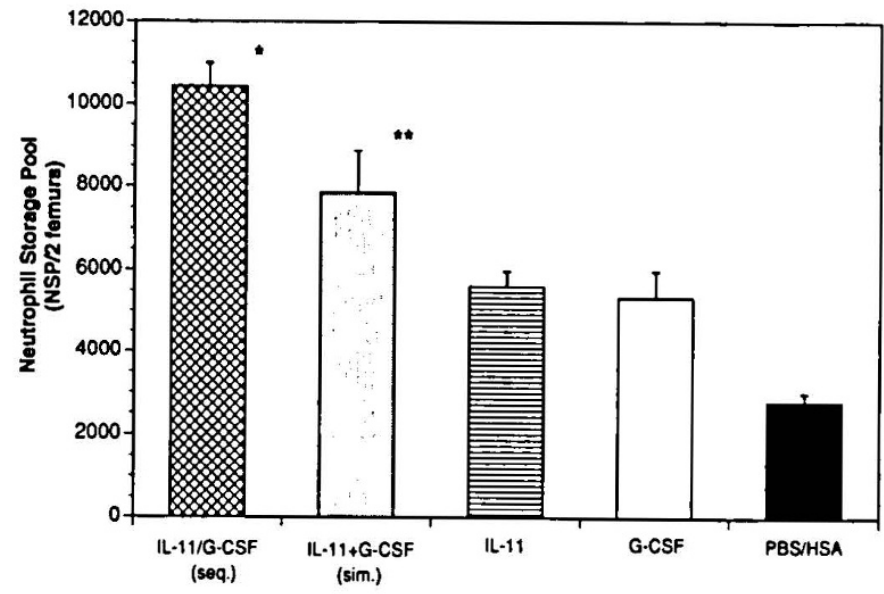

Fig. 6. Neonatal Sprague-Dawley rats $(\leq 24 \mathrm{~h})$ received rhIL-11 $\pm \mathrm{G}$ CSF for $14 \mathrm{~d}$, rhlL-11 for $7 \mathrm{~d}$ followed by G-CSF for $7 \mathrm{~d}$, or PBS/HSA for $14 \mathrm{~d}$ by daily intraperitoneal injection. BM NSP was determined by aseptic removal of the femurs and flushing of the bone marrow into a known quanity of Hanks' balanced salt solution. Electronic cell counts were performed and a 500-cell differential was obtained on Wright'sstained cytospin preparations. Bars represent the cell number per two femurs (mean \pm SEM) of three replicates from pooled samples of four animals per treatment group. ${ }^{*}$, rhIL-11 + G-CSF (sequential) vs G-CSF $(p<0.001)$ vs PBS/HSA $(p<0.03) ; * *$, rhIL-11 + G-CSF (simultaneous) vs G.CSF $(p<0.02)$ vs PBS/HSA $(p<0.001)$.

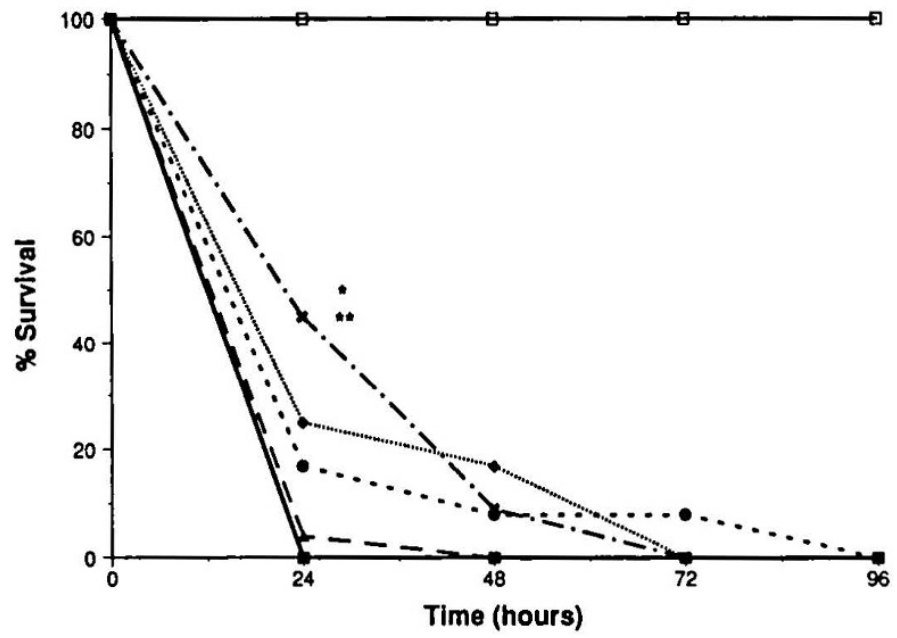

Fig. 7. Neonatal rats received rhIL-11, rhIL-11 and rhG-CSF sequentially or simultaneously, rhG-CSF, or PBS/HSA by intraperitoneal injection for $14 \mathrm{~d}$. Twelve animals per group were then inoculated with 2.5 $\times 10^{8} \mathrm{GBS} / \mathrm{g}$ on d 15 . Graph represents percentage of survival 0 to $96 \mathrm{~h}$ after injection of group B streptococcus. *, rhIL-11 + rhG-CSF (sequential) is rhG-CSF $(p<0.03)$; **, rhIL-11 + rhG-CSF (sequential) is PBS/ HSA $(p<0.04)$ at $24 \mathrm{~h}, 96 \mathrm{~h}(p=\mathrm{NS})$. no group B streptococcus: - . - IL-11 $\times 7 \mathrm{~d}+\mathrm{G}-\mathrm{CSF} \times 7 \mathrm{~d} ; \cdots$. . IL-11 + G-CSF $\times 14 \mathrm{~d}: \ldots$. . IL- $11 \times 14 \mathrm{~d}:-$. G-CSF $\times 14 \mathrm{~d}:-$. PBS/HSA $\times 14 \mathrm{~d}$.

survival at $24 \mathrm{~h}$ between IL-11 + G-CSF (sequential) versus GCSF $(p<0.03)$ and PBS/HSA $(p<0.04)$ (Fig. 7). However, by $96 \mathrm{~h}$, there was no significant difference in survival between any of the treatment groups ( $96 \mathrm{~h}, p=\mathrm{NS}$ ) (Fig. 7).

\section{DISCUSSION}

IL-11 has been found to synergize with IL-3 in supporting murine MEG colony formation and stimulating the proliferation of T-cell-dependent Ig-producing B cells (17). In addition to the synergistic effect of IL-11 with IL-3 to shorten the dormant $\mathrm{G}_{0}$ phase of stem cells, IL-11 has also been demonstrated to support 
the formation of CFU-GM, CFU-GEMM, and CFU-blast colony formation from cultures of marrow cells from normal mice (18). Recently, IL-11 was demonstrated to enhance CFU-GEMM formation when used in combination with Steel factor and IL-3 in serum containing cultures from d-2 enriched post-5-FU mouse marrow (15). Although IL-11 and IL-6 have similar in vitro biologic effects (i.e. shortened $\mathrm{G}_{0}$ dormant stem cell phase, synergistic with IL-3 on murine CFU-MEG proliferation, induced hepatic synthesis of acute-phase reactants, and stimulation of Ig B-cell production), they are truly two distinct cytokines (18).

Although total body neonatal rat concentrations of CFU-GM and NSP are significantly reduced compared with adult animals, the total concentrations of neonatal rat CFU-MEG are currently unknown (3-5). Recently, Olson et al. (20) demonstrated increased levels of circulating CFU-MEG in preterm and term cord blood compared with adult peripheral blood. These findings are similar to increased circulating levels of CFU-GM and CFUGEMM in preterm and term cord blood $(6,7)$. Thrombocytopenia $\left(<100000 / \mathrm{mm}^{3}\right)$ is commonly $(12 \%)$ found in preterm newborns $(<1500 \mathrm{~g})$ and is associated with an increased incidence of intraventricular hemorrhage compared with those preterm infants who have no evidence of thrombocytopenia (21).

Although administration of rhIL-11 alone had a negligible effect in neonatal rats on the circulating hematocrit and absolute neutrophil count, it induced a significant increase in the circulating platelet count ( 100 and $250 \mu \mathrm{g} / \mathrm{kg} / \mathrm{d})$. rhIL-11, however, also induced a significant increase in neonatal rat BM NSP, NPP, CFU-GM, and CFU-GM proliferative rates compared with placebo-controlled animals. The increase in the BM CFU-GM proliferative rate by rhIL-11 in vivo is similar to that demonstrated by Schibler et al., in vitro (19). However, the increase in BM myelopoietic precursors did not result in an increase in the circulating neutrophil count. It appears that secondary stimulation of committed myeloid progenitors and terminally differential myeloid cells with G-CSF is required before a peripheral response occurs.

When rhIL-11 was given both sequentially and simultaneously with rhG-CSF over a 14-d period, there was a significant increase in the circulating absolute neutrophil count during the $2 \mathrm{nd} w \mathrm{wk}$ of therapy compared with appropriate control-group animals. Additionally, the sequential and simultaneous administration of rhIL-11 and rhG-CSF induced a significant increase in the BM NSP over that of control animals and rhG-CSF alone. The simultaneous administration of rhIL-11 and rhG-CSF induced a significant increase in the BM NSP CFU-GM + CFU-GM proliferative rate similar to our previous studies using simultaneous rrSCF + rhG-CSF (12).

Although the combination of rhIL-11 and rhG-CSF induced a significant increase in the circulating absolute neutrophil count during the 2 nd wk of therapy, it failed to reduce the mortality rate during experimental group B streptococcal sepsis. We have previously demonstrated that $\mathrm{rSCF}$ alone or in combination with rhG-CSF, without antibiotics, reduced the mortality rate during experimental group B streptococcal sepsis. Because the combinations of rrSCF + rhG-CSF and rhIL-11 + rhG-CSF both demonstrated a significant increase in the circulating absolute neutrophil count and enhanced BM myeloid pools, other mechanisms besides augmentation of myelopoiesis may be playing a role in the difference in their prophylactic effect during experimental group B streptococcal sepsis (12). We postulate, as we previously demonstrated (12), that the increase in the BM mast cell pool by either rrSCF alone or in combination with rhG-CSF may play a role in enhancing neonatal rat host defense and thereby differentially reduce the mortality rate during experimental group B streptococcal sepsis. Alternatively, the differential individual and synergistic hematopoietic and host defense effects of IL-11 and SCF may also play a role in their relative ability to reduce morbidity and mortality during overwhelming bacterial infection. However, similar bacterial inoculums that induce a lower lethal dose at $24 \mathrm{~h}$ might benefit from IL-11 \pm G-CSF.

We have now demonstrated that both rhIL-11 and rhIL-6 enhance the circulating platelet count in neonatal rats (11). rhIL11 , however, either used alone or in combination with rhG-CSF, significantly induced a higher circulating platelet count compared with our previous rhIL-6 studies (11). Our study suggests that the in vitro effects of rhIL-11 on murine CFU-MEG proliferation also result in a significant in vivo response relative to an enhancement of the circulating platelet count. The in vivo effects of rhIL-11 on the circulating platelet count are similar to the in vivo effects of rhIL-6 $(22,23)$.

In summary, we have found that 14-d administration of rhIL11 in neonatal rats results in a significant increase in the circulating platelet count with relatively little effect on the circulating hematocrit and absolute neutrophil count. However, when rhlL11 was used either sequentially or simultaneously in combination with a lineage-specific CSF (rhG-CSF), there is enhancement in the circulating absolute neutrophil count. We hypothesize that rhIL-11 has more profound effects on neonatal thrombopoiesis compared with rrSCF or lineage-specific CSF such as rhG-CSF or recombinant murine GM-CSF. However, when rhIL-11 is used in combination with lineage-specific CSF, either sequentially or simultaneously, it may have additive effects on neonatal granulopoiesis by potentially shortening the dormant stem cell phase and inducing committed progenitor proliferation. rhIL-11 may have a potential role in either preventing or ameliorating thrombocytopenia in both preterm and term newborns.

Acknowledgment. The authors thank Linda Rahl for her expert editorial assistance in the preparation of this manuscript.

\section{REFERENCES}

1. Christensen R 1989 Hematopoiesis in the fetus and neonate. Pediatr Res 26:531-535

2. Cairo M 1989 Neonatal neutrophil host defense. Am J Dis Child 143:40-46

3. Castle V, Andrew M, Kelton J, Giron D, Johnston M, Carter C 1986 Frequency and mechanism of neonatal thrombocytopenia. J Pediatr 108:749-755

4. Christensen R, Rothstein G 1984 Pre- and post-natal development of granulocyte stem cells (CFUc) in the rat. Pediatr Res 18:599-602

5. Erdman SH, Christensen RD, Bradley PP, Rothstein G 1982 Supply and release of storage neutrophils: a developmental study. Biol Neonate 41:132137

6. Christensen RD, Macfarlane JL, Taylor NL, Hill HR, Rothstein G 1982 Blood and marrow neutrophils during experimental group B streptococcal infection: quantification of the stem cell, proliferative, storage, and circulating pools. Pediatr Res 16:549-553

7. Christensen R 1987 Circulating pluripotent hematopoietic progenitor cells in neonates. J Pediatr 110:622-625

8. Christensen R, Harper T, Rothstein G 1986 Granulocyte-macrophage progenitor cells in term and preterm neonates. J Pediatr 109:1047-1051

9. Cairo M, Mauss D, Kommareddy S, Norris K, van de Ven C, Modanlou H 1990 Prophylactic or simultaneous administration of recombinant human granulocyte colony stimulating factor in the treatment of group B streptococcal sepsis in neonatal rats. Pediatr Res 27:612-616

10. Cairo M, Plunkett J, Mauss D, van de Ven C 1990 Seven-day administration of recombinant human granulocyte colony-stimulating factor to newborn rats: modulation of neonatal neutrophilia, myelopoiesis, and group B streptococcus sepsis. Blood 76:1788-1794

11. Cairo M, Plunkett J, Nguyen A, Clark S, van de Ven C 1991 Sequential administration of interleukin- 6 and granulocyte colony-stimulating factor in newborn rats: modulation of newborn granulopoiesis and thrombopoiesis. Pediatr Res 30:554-559

12. Cairo M, Plunkett J, Nguyen A, van de Ven C 1992 Effect of stem cell factor with and without granulocyte colony-stimulating factor on neonatal hematopoiesis: in vivo induction of newborn myelopoiesis and reduction of mortality during experimental group B streptococcal sepsis. Blood 80:96101

13. Cairo M, Law P, van de Ven C, Plunkett J, Williams D, Ishizawa L, Gee A 1992 The in vitro effects of stem cell factor and PIXY321 on myeloid progenitor formation (CFU-GM) from immunomagnetic separated CD34 cord blood. Pediatr Res 32:277-281

14. Ikebuchi K, Wong G, Clark S, Ihle J, Hirai Y, Ogawa M 1987 Interleukin-6 enhancement of interleukin-3-dependent proliferation of multipotential hemopoietic progenitors. Proc Natl Acad Sci USA 84:9035-9039

15. Ikebuchi K, Clark S, Ihle J, Souza L, Ogawa M 1988 Granulocyte colonystimulating factor enhances interleukin-3-dependent proliferation of multipotential hemopoietic progenitors. Proc Natl Acad Sci USA 85:3445-3449 
16. Tsuji K, Zsebo K, Ogawa M 1991 Enhancement of murine blast cell colony formation in culture by recombinant rat stem cell factor (rrSCF), ligand for c-kit. Blood 78:1223-1229

17. Paul S, Bennett F, Calvetti J, Kelleher K, Wood C, O'Hara R, Leary A, Sibley B, Clark S, Williams D, Yang Y 1990 Molecular cloning of a cDNA encoding interleukin 11, a stromal cell-derived lymphopoietic and hematopoietic cytokine. Proc Natl Acad Sci USA 87:7512-7516

18. Musashi M, Yang Y, Paul S, Clark S, Sudo T, Ogawa M 1991 Direct and synergistic effects of interleukin 11 on murine hemopoiesis in culture. Proc Natl Acad Sci USA 88:765-769

19. Schibler K, Yang Y-C, Christensen R 1992 Effect of interleukin-11 on cycling status and clonogenic maturation of fetal and adult hematopoietic progenitors. Blood 80:900-903
20. Olson T, Levine R, Mazur E, Wright D, Salvado A 1992 Megakaryocytes and megakaryocyte progenitors in human cord blood. Am J Pediatr Hematol/ Oncol 14:241-247

21. Andrew M, Castle V, Saigal S, Carter C, Kelton J 1987 Clinical impact of neonatal thrombocytopenia. J Pediatr 110:457-464

22. Ishibashi T, Kimura H, Shikama Y, Uchida T, Kariyone S, Hirano T, Kishimoto T, Takatsuki F, Akiyama Y 1989 Interleukin-6 is a potent thrombopoietic factor in vivo in mice. Blood 74:1241-1244

23. Takatsuki F, Okano A, Suzuki C, Miyasaka Y, Hirano T, Kishimoto T, Ejima D, Akiyama Y 1990 Interleukin 6 perfusion stimulates reconstitution of the immune and hematopoietic systems after 5-fluorouracil treatment. Cancer Res 50:2885-2890 